\title{
MedienPädagogik
}

Zeitschrift für Theorie und Praxis der Medienbildung

\section{Räume erschliessen mit einem Smartphone im Sachunterricht an einer Grundschule}

\section{Möglichkeiten und Herausforderungen}

\author{
Stefanie Nickel
}

\section{Zusammenfassung}

Schulische Akteurinnen und Akteure, wie z. B. Lehramtsstudierende, haben häufig keine bzw. kaum frühkindliche Erfahrungen mit digitalen Medien, treffen jedoch auf eine nachwachsende Generation von Schülerinnen und Schülern, die bereits von klein auf in irgendeiner Weise in deren Nutzung eingebunden sind und entsprechendes Knowhow mitbringen. Lehr- und Lernprozesse beruhen auf dialogischer Gegenseitigkeit im Sinne einer Verschränkung von Subjekt und Struktur; es gilt daher, einen kooperativen und kommunikativen Erfahrungsraum beim Einsatz digitaler Medien zu schaffen. Um beispielsweise Räume mobil zu erschliessen, bietet sich das Erstellen einer Rallye auf dem Smartphone an. Auf diese Weise wird ein gemeinsamer Sachgegenstand aus dem Lebensumfeld der Schülerinnen und Schüler sowie der Studierenden aufgegriffen. Im Sinne des ausserschulischen Lernens erkunden und erschliessen sich die beteiligten Akteurinnen und Akteure gemeinsam im Team einen bestimmten Ort, um im Anschluss eine Rallye zu planen, auszuarbeiten sowie zu reflektieren. Im Fokus stehen dabei die Anbahnung und der Aufbau von Medienkompetenz durch den Umgang mit digitalen Medien, während im Arbeitsprozess durch dialogisch orientierte Aushandlungsprozesse in Kleingruppen heterogene Medienerfahrungen eingebracht werden. Zu fragen ist daher: Wie kann ein dialogisch angelegter Erfahrungsraum beim mobilen Lernen aussehen? 


\title{
Mobilize spaces with a rally via Smartphone. Possibilities and challenges
}

\begin{abstract}
School actors, such as teaching students, often have little or no early childhood experience with digital media, but meet pupils who are already involved in their use from an early age and have the necessary know-how. Teaching and learning processes are based on dialogical reciprocity in the sense of an entanglement of subject and structure. It is therefore important to create a cooperative and communicative space of experience in the use of digital media. In order to develop spaces on the move the creation of a mobile rally is an obvious choice. In this way, a common subject matter from the direct living environment of the pupils and students is taken up. In terms of out-of-school learning, the participating actors explore and develop a specific location together in the team in order to then plan, work out and reflect on a rally. In both groups the focus is on the initiation and development of media literacy through the use of digital media, while heterogeneous media experiences are introduced in small groups in the work process through dialogically oriented negotiation processes. The question is therefore: What can an experiential space look like, which offers the opportunity to learn mobilely?
\end{abstract}

\section{Vom Primat der überwältigenden Allmacht neuer Medien}

In seiner dystopischen Novelle 1984 schreibt George Orwell über die Macht gedanklicher Kontrolle durch Technik und über den Glauben daran, dass Realität nur im menschlichen Geist existiert (Orwell 1949). Mit Blick auf das Werk drängt sich die Frage nach der aktuellen Realität digitaler Medien und ihrem Einfluss sowohl auf die gesellschaftliche sowie kindliche Entwicklung auf. Orwells Erzählung zeichnet ein düsteres Bild von einer zukünftigen Gesellschaft, die unter massivem Einfluss technologischer und digitaler Wirkmechanismen steht und zu Machtzwecken ausgenutzt wird. Dies erfolgt auf Basis nicht hinterfragter Macht- und Hierarchieverhältnisse. Kurz gesagt: Der Roman beschreibt eine endgültige Abschiedserklärung 
an die Mündigkeit und Individualität des Einzelnen aufgrund technologischer und digitaler Allmacht. Die amerikanische Soziologin Sherry Turkle (2017) greift diesen Punkt in ihrem Buch Alone Together auf, personalisiert Technologie und macht sie zum «architect of our intimacies» (ebd., 1). Folgt man dem aktuellen öffentlichen Diskurs in sozialen Netzwerken, wiederholt sich der negativ urteilende, emotional aufgeladene Blick auf digitale Technik und Medien sowie ihre Nutzung durch Kinder - häufig einseitig.

Damit ist der Problemhorizont eröffnet: Eine allzu kritische, bewahrpädagogische Beurteilung digitaler Medien bis hin zur völligen Ablehnung verkennt Handlungsmöglichkeiten und räume. Diese gilt es ebenso zu reflektieren wie Herausforderungen und Risiken. Schon deshalb, weil sich bei der Implementation digitaler Medien in Lehr- und Lernprozesse etwas Neues in bestehende Strukturen einfügt und diese ebenso verändert. Neben Räumen braucht es daher ebenso Zeit, bis sich der Einsatz in organisationslogische, pädagogische und methodisch-didaktische Strukturen hineinentwickelt.

Dies stellt eine dialogische, ko-konstruktive und prozesshafte Gestaltungsaufgabe dar, an der Schülerinnen und Schüler, Eltern, Lehrerinnen und Lehrer, Pädagoginnen und Pädagogen sowie Lehramtsstudierende gleichermassen beteiligt sind, um u.a. beharrlichen Irrtümern (vgl. Spannagel 2015) zu begegnen und Widersprüche aufzulösen. Anders gesagt: Lehramtsstudierende haben häufig keine bzw. kaum frühkindliche Erfahrungen mit digitalen Medien gesammelt, treffen jedoch auf Schülerinnen und Schüler, die bereits von klein auf in irgendeiner Weise in digitale Kontexte eingebunden sind und entsprechendes Knowhow mitbringen (vgl. Wiesemann und Fürtig 2018). Prenskys (2001) Verständnis vom «digital native» verkennt jedoch, dass intuitives Handeln nicht gleich kompetentes Handeln ist. An dieser Stelle setzt das nachfolgende Projekt an. Denn dieses rückt das Zusammenspiel zwischen Theorie und Praxis im Lehramtsstudium in den Fokus sowie damit einhergehende Aktivitäten und strukturelle Gegebenheiten im Sinne einer Verschränkung von Subjekt und Struktur nach Anthony Giddens (1988). Thematisiert werden der Einsatz digitaler Technologien in der Grundschule sowie die gemeinsame Entwicklung von Ideen und didaktischen Konzepten mit Blick das Erschliessen von Räumen im Sachunterricht. Nachgezeichnet werden soziale Praktiken einer kleinen 
Kohorte von 50 Teilnehmerinnen und Teilnehmern im Umgang mit digitalen Technologien im Kontext der Ausgestaltung des Lehr- und Lernprozesses. Die Durchführung des Projekts erfolgte auf Basis des Selbstverständnisses von schulischer Aktionsforschung nach Altrichter, Posch und Spann (2018). Die Umsetzung wurde aus praxiserforschender Perspektive mittels Foto, Beobachtung, Interviews und Forschungstagebuch begleitet.

Aus forschungsrelevanter Perspektive ist also mit Blick auf die dialogische Verschränkung von Subjekt und Struktur zu fragen: Wie können kooperative und kommunikative Erfahrungsräume aussehen, in denen digitale Medien zum Einsatz kommen (Struktur)? Wie nutzen die schulischen Akteure und Akteurinnen die Angebote bzw. welche Einwände haben sie (Subjekt)? Und welche methodisch-didaktischen Überlegungen sind notwendig (Verschränkung von Subjekt und Struktur)? Um den Fragen in diesem Artikel nachzugehen, wird zunächst im zweiten Kapitel der Blick auf die sich verändernde Lebenswelt der Kinder und jungen Erwachsenen gerichtet. Im Anschluss erfolgen im dritten Kapitel eine skizzenhafte Begriffsklärung sowie eine darauf aufbauende Auseinandersetzung mit einem Modell zur Gestaltung von Lehr- und Lernprozessen mit digitalen Medien. Bei diesem wird ein dialogisches Verhältnis zwischen Medienkompetenz und Medienbildung hergeleitet. In diesem zeigen sich Zusammenhänge zwischen medienpädagogischen sowie Grundschul- und Sachunterrichtsdidaktischen Überlegungen. Auf dieser Grundlage findet im vierten Kapitel eine Auseinandersetzung mit Möglichkeiten der Raumkonstruktion und -aneignung statt, um die Relevanz des Einbezugs von ausserschulischen Lernorten im konkret-physischen Raum mit Blick auf das Lernen in der Grundschule zu verdeutlichen. Mit Hilfe eines Praxisbeispiels erfolgt im fünften Kapitel der Versuch einer Theorie-Praxis-Verzahnung im Lehramtsstudium.

\section{Mediatisierung der Lebenswelt}

Für die Hinwendung zu einer Unterrichtsgestaltung (vgl. Irion 2018; Irion 2016; Peschel 2016) mit digitalen Medien spricht die in der gegenwärtigen Zeit aktive und breite Nutzung dieser durch Kinder und (junge) Erwachsene in ihrem gesellschaftlichen und durch historische Entwicklungen 
geprägten Lebensraum. Dieser fungiert als Basis für ihre Entwicklung und Bildung. Unter dem Konzept der mediatisierten Kindheit (vgl. Tillmann und Hugger 2014) wird verstanden, dass digitale Medien ein «selbstverständlicher Bestandteil der kindlichen Lebens- und Alltagswelt» (ebd., 31f.) sind. Das heisst, technische Aspekte sowie kommunikative, kulturelle und soziale Praktiken verändern sich durch die Nutzung. Anders gesagt: Digitale Medien wirken sich auf das Gestalten von Beziehungen, Mobilität und Aushandlungsprozesse zwischen Kindern und Erwachsenen (vgl. ebd.) aus. Kinder und Erwachsene als soziale Akteurinnen und Akteure gestalten und verändern ihre Umwelt aktiv durch ihre alltäglichen Praktiken. Zugleich entwickeln sie durch ihr Handeln ihre Persönlichkeit im Kontext eines medial geprägten Lebensraums. Dieser soziale Raum umfasst nach Baacke (vgl. 1998) alle Kommunikationen der miteinander agierenden Menschen. Aus diesem Grund wird Medienkompetenz ein zentraler Stellenwert zugesprochen. Digitale Medien wirken auf die Lebensgestaltung in diesem Raum. Sie tragen zu kulturellen und sozialen Wandlungsprozessen im Rahmen gesellschaftlicher Praktiken, bei der Ausgestaltung von Beziehungsstrukturen sowie zur individuellen und kollektiven Identitätsbildung bei (vgl. Fürtig und Wiesemann 2018; Irion 2018; Aufenanger 2015; Spannagel 2015; Tillmann und Hugger 2014; Spanhel 2010; Tulodziecki und Herzig 2010).

Beispielsweise zeigt der Blick auf aktuelle Studien, dass 99\% der 13-19-Jährigen ein Smartphone besitzen und zur Freizeitgestaltung und Informationsbeschaffung nutzen (vgl. Feierabend, Plankenhorn, und Rathgeb 2016). Bei den 6-13-Jährigen zeigen zwei Drittel Interesse an den Themengebieten «Handy/Smartphone» und «Internet/Computer/Laptop». 53\% der befragten Kinder zwischen 6 und 13 besitzen bereits ein Smartphone und nutzen dieses regelmässig. Zu den beliebtesten Plattformen gehören WhatsApp, Youtube, Facebook, Instagram und Snapchat (vgl. Feierabend, Plankenhorn, und Rathgeb 2017).

Neben dem Bezug zum Lebensraum sprechen daher auch zukünftige Entwicklungen im digitalen Kontext sowie eine Unterstützungsfunktion, die digitale Medien beim Lernen einnehmen können (Döbeli Honegger 2016). Eine sachgemässe, kreative und reflektierte Einbindung in universitäre wie auch schulische Lehr- und Lernprozesse erscheint relevant. Für 
die weitere Auseinandersetzung mit der Thematik rückt daher zunächst die Klärung der Begriffe Medien, Medienkompetenz und Medienbildung in den Fokus. Aus den nachfolgenden Gedanken wird im Anschluss ein Modell abgeleitet, um Handlungsmöglichkeiten sowie strukturelle Implikationen aufzuzeigen.

\section{Medien, Medienkompetenz und Medienbildung}

Tulodziecki und Herzig (2010) verstehen Medien als Mittel bzw. etwas Vermittelndes, «durch die in kommunikativen Zusammenhängen potentielle Zeichen mit technischer Unterstützung» (ebd., 18) genutzt werden. Das Verständnis von Medien als reine Mittler greift zu kurz, da Medien ebenso umwelt- und strukturprägend sind. Spanhel (2010) verweist in diesem Zusammenhang darauf, dass Medien auch als «Träger von Kultur und Gesellschaft» verstanden werden sollten (vgl. ebd., 50). Die bei Spanhel angesprochene Zeichenfähigkeit ermöglicht dem Subjekt Handlung und Reflexion im Kontext von strukturellen Rahmenbedingungen. Diese Perspektive eröffnet neue Handlungsmöglichkeiten und -räume für das Subjekt ${ }^{1}$. In Anlehnung an die enge Definition von Pirnay-Dummer und Spengler (2019) werden Medien im Folgenden daher verstanden als Mittler und Vermittler, wodurch der pädagogisch Handelnde zu einem Primärmedium wird, während Materialien als Sekundärmedium fungieren und angewandte Methoden der Vermittlung von Wissen als Tertiärmedien (ebd., 483). Eine ähnlich enge Definition liefert auch Moser (2019, 1 ff.).

So wird in diesem Artikel unter Medienkompetenz die Anwendung erlernter Muster verstanden (vgl. Spanhel 2010). Operativ heisst das für das Vermitteln und Aneignen von Medienkompetenz im institutionellen Kontext: Um Medien kompetent zu nutzen, eignet sich das Subjekt im Sinne Weinerts (2001) entsprechendes Wissen, Können und Haltungen zu und über Strukturen an (Tulodziecki 2019, 199 ff. u. 2011, 23; zum Medienkompetenzbegriff siehe auch Ladel, Knopf, und Weinberger 2018, 95 ff.).

1 In Orwells 1984 beispielsweise dient ein Tagebuch als Medium und wird zum Gegenstand einer heimlich geführten Auseinandersetzung zwischen Mensch und Umwelt. Es stellt dabei sowohl den Vermittler einer kommunikativen Praktik dar (Zeichen schreiben) sowie den Träger einer sozio-kulturellen Reflexion (Zeichen deuten und verstehen) des Individuums. 
Medienbildung meint demgegenüber hier in Anlehnung an Spanhel (2014, 587) einen (lebenslangen) Prozess der Auseinandersetzung mit Medien sowie das Ergebnis von Reflexion über Medien. Das heisst, das Subjekt verändert aktiv und eigenständig Deutungs- und Handlungsmuster, um ein aufgeschlossenes Verhältnis zwischen sich und seiner Umwelt zu entwickeln. Hierin zeigt sich ein transformativer Bildungsbegriff.

Mit Blick auf die unterschiedlichen Definitionen kann gefolgert werden, dass beiden Begriffen der prozesshafte und reflexive Moment gemein ist. Eine konkurrierende Gegenüberstellung wird demnach obsolet. Sie können vielmehr dialogisch gedacht werden. Das heisst, der Kompetenzbegriff fokussiert sich auf strukturelle Rahmenbedingungen sowie auf funktionale, strukturierende und messbare bzw. normative Aspekte. Diese können als Zielgrösse in Lehr- und Lernprozessen geltend gemacht werden. Der Kompetenzerwerb bildet demzufolge eine Brücke für das Subjekt hin zu veränderbaren Rahmenbedingungen sowie zu einer umfassenden Persönlichkeitsentwicklung. Im Bildungsbegriff hingegen zeigen sich subjektorientierte, flexible und wertfreie Aspekte. Sie sind nicht messbar. Bildungsprozesse stellen vielmehr einen transformativen und individuellen Akt der Selbst- und Weltaneignung dar, mit dem Ziel der persönlichen Entfaltung von Anlagen (vgl. Klafki 1959, 180f.).

In Anlehnung an Giddens (1988) lässt sich daher folgendes Modell für die Gestaltung von (hoch-)schuldidaktischen Erfahrungsräumen unter Berücksichtigung von digitalen Medien zugrunde legen (siehe Abb. 1). 


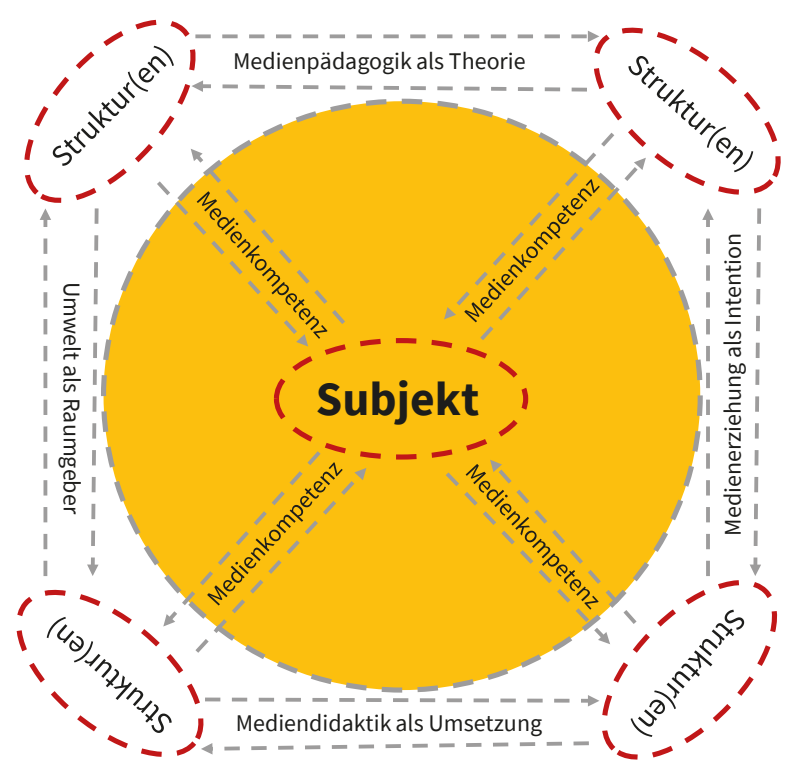

Abb. 1.: Eigene Darstellung in Anlehnung an A. Giddens Strukturationstheorie (vgl. 1988).

Strukturelle Bedingungen resultieren u.a. aus Zielsetzungen (siehe z. B. Strategiepapier der KMK) sowie theoretischen Grundlagen und technischen Aspekten über digitale Medien. Inhaltlich sollten sich Studierende daher gezielt mit diesen auseinandersetzen und den Sachgegenstand in aktuelle Diskurse einbetten. Ziel ist dabei, dem intuitiv gesteuerten Erfahrungsvorsprung der Kinder und Jugendlichen im Schulalltag zu begegnen. In diesem Zusammenhang konstatiert Peschel (2016, 45f.), das Ziel, Inhalt und Methode genau festgelegt und das didaktische Setting mit Sorgfalt geplant werden sollten, um die eingangs erwähnte schwarz/ weiss-Zeichnung nicht noch weiter zu begünstigen. Aus hochschuldidaktischer Perspektive sollte aus diesem Grund den Studierenden ein kritischreflektierter Umgang mit und durch digitale Medien nähergebracht werden. Dies erfolgt, indem ein Sachgegenstand (z. B. Smartphone, Tablet und Apps) aufgegriffen und inhaltlich an die Erschliessung im konkreten Raum angebunden wird. Wissenschaftlich-reflexiv und institutionell nachhaltig verorten lässt sich die Gestaltung des Lehr- und Lernsettings daher als Erkenntnisgewinnung unter praxiserforschender Perspektive: Sie erfolgt durch die Verzahnung von Theorie-Praxis im Lehramtsstudium, weil Studierende sowie Schülerinnen und Schüler gemeinsam im Team arbeiten. 


\section{Räume, Raumverschränkung und ausserschulische Lernorte}

Aus den vorangegangenen Gedankengängen lässt sich schliessen, dass strukturelle Bedingungen veränderbar sind. Durch einen kontinuierlichen Strom von Handlungen konstruiert das Subjekt den sozialen Raum und verändert ihn fortlaufend. Martina Löw $(2011,85 f$.) legt beispielsweise eine Raumvorstellung zugrunde, bei der die Konstitution von sozialen Räumen in zweierlei Hinsicht erfahren wird: Zum einen als einheitliche Inseln und zum anderen als heterogener, uneinheitlicher Raum. Zentral an Löws Ausführungen zum Raum ist in diesem Kontext, dass der Raumwandel durch «Erfahrungen in virtuellen Räumen» unterstützt wird (ebd., 86). Durch diese Annahme lassen sich unterschiedliche Vorerfahrungen, Kenntnisse und Präferenzen erklären - auch mit Blick auf entsprechende Sozialisationsprozesse. Der virtuelle Raum bezieht sich hier auf Vorstellungen aus gemachten Erfahrungen, d.h. auf die rein geistige Realität des Subjekts. Es handelt sich um einen nicht greif- und fassbaren Ort, der nicht konkret erfahrbar ist und eine andere Zeittaktung aufweist. Betrachtet man z. B. die Handlungen virtueller Personen (der Sprung eines Avatars von einer Plattform in die Tiefe), haben diese keine Auswirkungen auf den Spieler.

Ein Blick auf Untersuchungen von Jean Piaget zur Aneignung des räumlichen Vorstellungsvermögens erscheint interessant, um weitere Schlüsse für das Gestalten von Lehr- und Lernprozessen mit digitalen Medien zu ziehen. Als entwicklungspsychologisch fortschreitender Aneignungsprozess verstanden, begreift Piaget (1982) im Rahmen seiner Untersuchungen diesen auf zwei Ebenen: Wahrnehmung und Vorstellung. Kennzeichnend für das Verständnis ist das dialogische Verhältnis zwischen den Ebenen. Wahrnehmung meint das konkrete Erkennen von Gegenständen, während die Vorstellung der Gegenstände sich im Geiste vollzieht (vgl. Piaget 1982). Mit anderen Worten: das eine braucht den direkten, physischen Kontakt, das andere baut auf bereits vorhandenen Wissensbeständen durch den physischen Kontakt auf. Dieser Prozess findet quasi virtuell statt, d.h. rein geistig. Durch Handlung entwickelt sich demnach räumliches Denken (vgl. ebd., 75ff).

In der Alterspanne zwischen sieben bis ca. 12 Jahren befinden sich Kinder Piaget zufolge in der konkret-operationalen Phase. Im Rahmen ihres 
kognitiven Erkenntnisprozesses ist ihr Handeln auf Gegenstände ausgerichtet, um vorhandene Denk- und Handlungsschemata zu verändern bzw. anzupassen oder aufzubauen. Grundlegende Voraussetzung für die Entwicklung des Denk- und Erkenntnisvermögens ist die tätige Auseinandersetzung mit dem Lebensraum, das Streben nach einem Ausgleich zwischen Strukturen und ihren Inhalten sowie der Reifegrad der jeweils physiologischen Anlagen. Piagets Theorie passt zu einem konstruktivistisch angelegten Lehr- und Lernverständnis. ${ }^{2}$

Zusammenfassend lässt sich sagen: Handlungen führen zu Erfahrungen, die wiederum auf Wahrnehmungen gründen und zu reflektierten Vorstellungen werden können. Bei Baar und Schönknecht (2018) werden Erfahrungen als «Ausgangspunkt von Lernen, Wissen und Bildung» (ebd., 7) verstanden. Sie können zu nachhaltigen Bildungserkenntnissen verhelfen, wenn sie in «konkreten Situationen in Form von subjektiv sinnbezogenem, entdeckendem und selbstbestimmten Lernen gemacht werden» (ebd.). Das Wahrnehmen und Erfahren des Fassbaren und Gegenständlichen im konkret-physischen Raum ist für die Lernprozesse von Kindern im Grundschulalter unerlässlich. Die Erfahrungen unterstützen dabei, Weltanschauungen aufzubauen, kritisch zu hinterfragen und autonom zu reflektieren. Das heisst, es geht um das bewusste Ein- und Aussteigen in unterschiedliche Erfahrungsräume.

Aus diesem Grund wird im vorliegenden Artikel die Unterscheidung zwischen realem und virtuellem Raum bzw. physischer und geistiger Realität aufrechterhalten. Mit zunehmender medialer Durchdringung der frühkindlichen Lebenswelt verlieren die Grenzen jedoch an Konturen, sodass Räume nur durch die Reflexion derselben als getrennt wahrgenommen werden (hierzu siehe u.a. Koller 2018, 274-282). Die Unterscheidung erfolgt hier dennoch, um Möglichkeiten, Bedingungen und Grenzen der unterschiedlichen Raumstrukturen transparent zu machen. Darauf bezogen kann das Handeln der Subjekte mit Fokus auf Identitätsbildung,

2 Löw kritisiert jedoch zu Recht, dass bei Piagets Ausführungen zum kognitiven Lernprozess kulturell tradierte Raumvorstellungen durch entsprechende Sozialisationsprozesse nicht berücksichtigt werden (vgl. Löw 2011, 76f.). Die sozialökologischen Ansätze von Uli Bronfenbrenner (1981) und Dieter Baacke (1993) liefern Aufschluss. Sie besagen, dass sich Kinder und Jugendliche Räume im Sinne konzentrischer, immer grösser werdender Kreise erschliessen. 
persönliche Relevanzsetzungen und biographische Hintergründe reflektiert werden. Dies erfolgt vor der Annahme Prenskys (2001), dass Kinder möglicherweise digitale Medien aufgrund der frühen Einbettung eher intuitiv nutzen und Räume als zusammengefasst wahrnehmen, während sich Erwachsene entsprechendes Knowhow nach und nach aneignen und Räume eher als getrennt verstehen (vgl. Koller 2018). Ob und wie sich das zeigt, wird im nachfolgenden Praxisbeispiel aufgezeigt.

\section{Räume mobil erschliessen mit dem Smartphone}

Um Räume mobil zu erschliessen und sich Vorstellungen darüber bewusst zu machen, bietet sich das Erstellen einer Rallye auf dem Smartphone an. Auf diese Weise wird ein Sachgegenstand aus dem Lebensumfeld der Schülerinnen und Schüler sowie Studierenden aufgegriffen. Im Sinne des ausserschulischen Lernens entdecken, erkunden und stellen die beteiligten Akteurinnen und Akteure im Team Fragen zum ausgewählten Lernort, um im Anschluss eine Rallye zu planen, auszuarbeiten sowie um gemeinsam zu reflektieren. Im Fokus des Projekts stehen insbesondere auf Seiten der Studierenden die Anbahnung und der Aufbau von kommunikativer Kompetenz sowie von situativer Handlungspraxis im Umgang mit digitalen Medien. Entsprechende Kompetenzen sollen bei den Schülerinnen und Schülern sowie den Studierenden aus den in der KMK (2016) zugrunde gelegten sechs Kompetenzbereichen (ebd., 15 ff.) angebahnt werden. Darunter fallen im Einzelnen:

Arbeitsinteressen klären und festlegen; digitale Kommunikationsmöglichkeiten zielgerichtet auswählen; digitale Werkzeuge für die Zusammenarbeit bei der Zusammenführung nutzen; Verhaltensregeln bei digitaler Interaktion kennenlernen und die Kommunikation anpassen; öffentliche Dienste nutzen; Inhalte in verschiedenen Formaten bearbeiten, zusammenführen und veröffentlichen/teilen; Inhalte in bestehendes Wissen integrieren; Bedeutung von Urheberrecht und Gestaltungsmittel von digitalen Medienangeboten kennenlernen; Chancen und Risiken des Mediengebrauchs reflektieren und Potenziale erkennen; technische Probleme identifizieren und Lösungen ermitteln. 
Aus diesem Grund richtet sich der Blick ganz konkret auf zwei Ebenen:

1. Auf Lehramtsstudierende in der ersten Phase der Lehramtsausbildung mit dem Ziel der Sensibilisierung für Lehrerprofessionalisierung und Reflexion der eigenen Lehrerprofessionalität sowie Anbahnung von Kompetenzen bezogen auf das Handlungsfeld unter besonderer Berücksichtigung von digitalen Technologien in der Grundschule.

2. Auf Schülerinnen und Schüler der Grundschule mit den Ziel der Anbahnung von Medienkompetenz und Sensibilisierung für kritische Fragestellungen über das Nutzen von digitalen Medien sowie Erarbeiten eines Grundverständnisses für Logiken des Programmierens.

Im Prozessverlauf erkundeten die Schülerinnen und Schüler sowie die Studierenden mechanische Funktionen an technischen Gegenständen, um die Abläufe beim Erstellen einer Rallye selbsttätig nachzuvollziehen. Das Pilotprojekt ${ }^{3}$ «Future-LAB - Digitales Lernen» zielte dabei auf eine nachhaltige Auseinandersetzung mit dem fachvernetzenden Themenbereich digitale Medien $\mathrm{ab}^{4}$. Gestartet wurde im April 2018 in Zusammenarbeit mit einer Grundschule in Siegen. Das Besondere der beteiligten Glückauf Grundschule ist ihre inklusive Ausrichtung sowie ihre jahrgangsübergreifenden Klassen, die altersgemischten Lerngruppen und die überaus ansprechenden, materialreichen Klassenräume. Die Schule hat sich zum Ziel gesetzt, die Vielfalt der gemeinsamen Gruppen zu nutzen und jedes der insgesamt 205 Kinder auf seinem individuellen Weg zu begleiten; hierfür stehen Integrationskräfte helfend zur Seite.

3 Siehe Beitrag mit Interview im WDR Lokalzeit Südwestfalen: «Kinder entwickeln App». (Gekürzte Version: https://wwwl.wdr.de/mediathek/video/sendungen/lokalzeit-suedwestfalen/to-go/video-kinder-entwickeln-app--10o. html).

4 Das hier vorgestellte Beispiel zum Erschliessen von Räumen mit einer Smartphone-Rallye sowie die daraus gewonnenen Erkenntnisse basieren auf einer kleinen Kohorte von insgesamt knapp 50 teilnehmenden Schülerinnen und Schülern sowie Studierenden. Eine Generalisierung der Ergebnisse ist daher nicht möglich. In diesem Beitrag geht es vielmehr um das Vorstellen eines theoretischen Modells sowie das darauf aufbauende methodisch-didaktische Umsetzen in der Praxis im Sinne einer dialogischen Verschränkung von Subjekt und Struktur. 

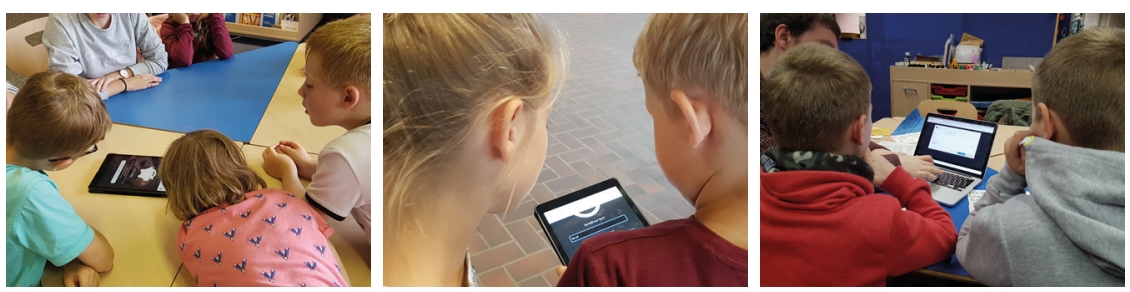

Abb. 2.: Studierende der Universität Siegen sowie Schülerinnen und Schüler der Glückauf Grundschule in Siegen beim Programmieren und Spielen einer Rallye mit dem Smartphone.

Zunächst galt es, eine theoretische Grundlage im projektbegleitenden Seminar «Räume mobil erschliessen mit einer Smartphone-Rallye» zu legen. Als Basis diente hierfür u.a. das weiter oben dargelegte Modell zum dialogischen Zusammenhang von Subjekt und Struktur (siehe Abb. 1). Dieses wurde in der Eröffnungssitzung vorgestellt sowie einschlägige schul- und medienpädagogische Diskurse bearbeitet. Der Themenbereich Digitales Lernen wurde auf diese Weise exemplarisch genutzt, um gemeinsam mit den Studierenden die Herangehensweise und Handhabe mit Webinhalten und Social-Networking aufzubereiten und zu erörtern. Das heisst, es ging um eine analytisch-reflexive und ethische Auseinandersetzung, mit dem Ziel einer kritischen Betrachtung der eingesetzten Medien (Ladel, Knopf, und Weinberger 2018). Zur Disposition standen u.a. Fragen mit Blick auf etwaige Bewältigungsstrategien, die zu einer Orientierung im Netz beitragen, wie zum Beispiel: Darf ich Bilder online stellen und wenn ja, welche? Was muss ich beachten? Wer kann meine Inhalte sehen? Welche Probleme können auftreten und wie gehe ich mit diesen um?

Unter Rückgriff auf die weiter oben skizzierten Begründungszusammenhänge zwischen Lebensraumbezug, Zukunftsbedeutung und Lernunterstützung wurde die gegenwärtige Bedeutung des Themenbereichs diskutiert (Döbeli Honegger 2016). Als zukunftsrelevant sahen die Studierenden dabei das Einbinden digitaler Medien mit Blick auf aktuelle Trends im komplexen Sozialgefüge von Online-Plattformen. Diese werden häufig von Kindern im Grundschulalter genutzt; neue Sprachkulturen und Berufsfelder gehen daraus hervor. Den eher selbstverständlichen Umgang der Kinder grenzten die Studierenden ab von einer fundierten Kenntnis über Auswirkungen, Grenzen und Gefahren der Nutzung (Irion 2018). Hierunter fiel beispielsweise, dass Kinder immer häufiger eingebunden werden 
als ökonomische Zielgruppe, weil sich gesellschaftliche Kontexte im Netz überschneiden. Mit Blick auf die eingangs erwähnten Haltungen gegenüber digitalen Medien sowie ihre Nutzung durch Kinder zeigte sich in der Auseinandersetzung unter den Studierenden ein Spannungsbogen aus kritisch-bewahrpädagogischer und fasziniert-euphorischer Beurteilung. Dazu schreibt eine Studentin in ihrem Forschungstagebuch:

«Ich hatte vor dem Seminar eine sehr kritische Haltung gegenüber digitalen Medien. Ich finde, dass der Konsum dieser in den letzten Jahren, besonders bei Kindern und Jugendlichen, aber auch bei Erwachsenen, gestiegen ist. Kaum jemand kann sich eine Welt ohne die digitalen Medien vorstellen. Jeder benutzt Smartphones, soziale Medien (Facebook, Instagram, Snapchat etc.) und auch Laptops, Tablet, Spielkonsolen und Fernseher fehlen in kaum einem Haushalt. Aufgrund dessen hatte ich eine sehr negative Haltung gegenüber digitalen Medien. Ich bin der Meinung, dass gerade in der Grundschule nicht der Fokus auf den digitalen Medien liegen sollte, da die Kinder schon genug Zeit mit diesen außerhalb der Schule verbringen» (vgl. FtStud1_w, S. 14).

Es zeigte sich ferner ein überaus heterogener Kenntnis- und Fähigkeitsstand im Hinblick auf technische Aspekte; wenngleich alle teilnehmenden Studierenden seit ihrem ca. 13. Lebensjahr ein eigenes Smartphone besitzen sowie Tablets, Laptops, entsprechende Programme und Apps nutzen - teilweise überaus kompetent. In diesem Zusammenhang sollte mit Hilfe des Projekts der vermittelnde Aspekt von Medien greifbar gemacht werden, indem sich die Studierenden sowohl informativ als auch instrumentell-qualifikatorisch mit diesen auseinandersetzen. Der nächste Schritt bestand daher darin, dass sich die Studierenden die Funktionsweise der App Actionbound aneigneten. Hierfür bot die Lernwerkstatt OASE an der Universität Siegen optimalen Raum zur Erkundung. Um sich das Programm zu erschliessen, spielten die Studierenden eine vorbereitete Rallye mit zur Verfügung gestellten Tablets. Auf diesen war die App bereits installiert. Im Anschluss wurden einzelne Funktionen via Beamer-Projektion schrittweise erläutert. Es galt zu klären, was unter der Actionbound-App zu verstehen ist und wie sie funktioniert. 
Bei der hier ausgewählten App handelt es sich um eine relativ einfach zu bedienendes Programm, das über eine Dozenten-Lizenz beim Anbieter für die Anwendung in Seminaren erworben werden kann. Früher besser bekannt als via paper und pencil erstellte Schnitzeljagd, wird diese Funktion nun durch das Nutzen von digitalen Fotos, Filmen und GPS via Smartphone und Tablet erweitert. Auf «www.actionbound.com» wird ein Account erstellt, auf dem sich die die Nutzer einloggen. Die Rallye bekommt einen Namen und der Inhalt wird festgelegt. Das heisst, entsprechende Aufgaben werden formuliert. So kann die Jagd zum Beispiel mit dem Finden eines Ortes über GPS beginnen. Dies ist wichtig, denn die Spieler sollten wissen, wo die Rallye anfängt. Es gilt also, zunächst einen Startpunkt festzulegen. Im Anschluss bietet die App die Möglichkeit, Abschnitte festzulegen, Infos bereit zu stellen sowie Quizze und Aufgaben zu formulieren sowie weitere Koordinaten zu bestimmen. Ziel ist es, genau wie bei der Schnitzeljagd, Rätsel und Aufgaben zu lösen, um möglichst viele Punkte zu sammeln und am Ende erfolgreich den Endpunkt zu erreichen - möglichst als Erster. Die Spieler werden auf diese Weise u.a. zu Reportern, Entdeckern und Erkundern.

Bevor die Arbeit mit den Schülerinnen und Schülern begann, programmierten die Studierenden zunächst eigenständig kleinere Bounds, um die Funktion der App zu durchdringen. In einer weiteren Sitzung galt es dann, die Herangehensweise methodisch-didaktisch für Grundschülerinnen und -schüler aufzubereiten. Dies erfolgte vor dem Hintergrund der weiter oben dargelegten Annahmen über den Raum (siehe 4. Kapitel). Gefragt wurde, wie das Erstellen einer Rallye auf dem Smartphone Kindern im Grundschulalter nähergebracht werden kann. Mit Hilfe des didaktischen Modells nach Klafki, legten die Studierenden den Ablauf der gemeinsamen Treffen mit den Schülerinnen und Schülern fest und setzten sich mit theoretischen Aspekten auseinander: Ziele, Methoden und Inhalte sowie Gegenwarts-, Zukunfts- und exemplarische Bedeutung des Sachgegenstands wurden ausgelotet und festgehalten sowie mögliche Bedenken diskutiert. Hierbei lag der Fokus auf einer innovativen und kreativen Ausgestaltung der eingesetzten Medien. 
Die Auseinandersetzung fand in Kleingruppen statt; die Gruppenzusammensetzung blieb über den gesamten Prozessverlauf bestehen. Diese Vorgehensweise hat sich im weiteren Verlauf bewährt, da die einzelnen Kleingruppen aus maximal sechs Personen bestanden (im Verhältnis zwei Studierende zu vier Schülerinnen bzw. Schülern). Dies ermöglichte ein direktes Einlassen auf die heterogene Gruppenzusammensetzung. In diesen waren je unterschiedliche Vorerfahrungen und Kenntnisstände mit und über digitale Medien vorhanden. Die Studierenden planten die einzelnen Sitzungen eigenständig und bereiteten die Umsetzung gemäss ihrer Kleingruppe didaktisch vor und nach. Auf diese Weise erweiterten sie ihr eigenes situatives Handlungsrepertoire, da auf Situationen direkt reagiert werden musste. So entstanden Erklärfilme, Karten und Poster, um die Funktionsweise sowie die Symboliken der App für die Schülerinnen und Schüler konkret sichtbar zu gestalten. In diesem Vorgehen steckt der Zusammenhang zwischen Subjekt und Struktur sowie der im dritten Kapitel dargelegte Medienbegriff. Für die didaktische Gestaltung und das Kennenlernen der Schülerinnen und Schüler standen zwei Sitzungen zur Verfügung.
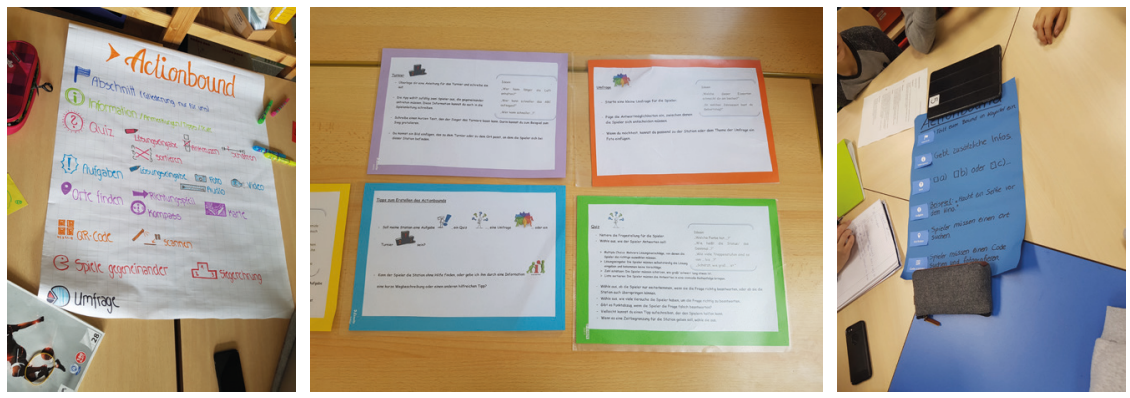

Abb. 3.: Didaktisches Material der Studierenden im Seminar.

Um mit den Schülerinnen und Schülern eine virtuelle Rallye-Strecke festzulegen, war der Besuch von konkret-physischen Orten notwendig. Das heisst, ausserschulische Lernorte wurden besucht. Hierfür kamen kulturelle Orte in Siegen (z. B. Theater, Museum, Bibliothek) ebenso wie Orte der Natur (z. B. Parkanlagen) und des gesellschaftlichen Zusammenlebens (z. B. bestimmte Stadtviertel bzw. die Oberstadt) in Frage. Als Lernorte ohne Bildungsauftrag (Baar und Schönknecht 2018, 21) stellen sie zudem im Sinne Michel Foucaults (1992) Heterotopien dar. Sie funktionieren auf 
Basis vorgegebener Normen und Regeln mittels entsprechender Ressourcen; durch die intentionale Einbindung in Lehr- und Lernprozesse wurden diese anderen Räume (ebd.) zu ausserschulischen Lernorten. Die in den Kleingruppen organisierte Exkursion diente schliesslich dazu, die RallyeStrecke zu organisieren, Bilder zu machen, Zeiträume festzulegen und Informationen am konkret physischen Ort einzuholen. Dazu schreibt eine Studentin in ihrem Forschungstagebuch:

«Die Schüler und Schülerinnen waren sehr aufgeregt, dass wir das Projekt mit i-Pads durchführen werden. (...) Beim Aufnehmen der Fotos war dann irgendwann der Speicher voll und wir waren überfordert das i-Pad wieder zum Laufen zu bringen. Eine Schülerin hat unsere Überforderung wahrgenommen und konnte uns sofort erklären, wie wir die Bilder löschen und dass wir das i-Pad neustarten müssen, um wieder Fotos aufnehmen zu können. Hier hat sich gezeigt, dass Kinder im Umgang mit Medien oft fitter sind als wir.» (FtStud7_w, o.S.).

Die Verschiedenheit der Orte führte dazu, dass die einzelnen Gruppen unterschiedliche Erfahrungen, Fotos, Fragen und Antworten sammelten, die im Anschluss in der App actionbound zusammengeführt werden sollten. Das systematische Erkunden mit Fokus auf das Erstellen einer zusammenhängenden Rallye-Strecke hat die Kinder dabei unterstützt, sich ihr direktes Lebensumfeld zu erschliessen. Mit anderen Worten: Sie konnten sich durch aufkommende Fragen mit unterschiedlichen Facetten der Orte vertraut machen und eine konkrete Vorstellung aufbauen. Diese wurde rückwirkend reflektiert. Das Tablet wurde dabei als Erinnerungshilfe für die rückwirkende Vorstellung beim Programmieren genutzt. An den einzelnen Orten eröffneten sich eine Reihe ungeplanter und beiläufiger Lernmöglichkeiten, im Sinne des informellen Lernens (vgl. Baar und Schönknecht 2018, 16). Beispielsweise konnten die Schülerinnen und Schüler in der Lernwerkstatt an der Universität ungestört einzelne Gegenstände (z. B. Waage, div. Instrumente, Bücher) ausprobieren, testen und verfremden sowie eigene Handlungsrelevanzen bei der Aufgabenentwicklung setzen und die Realisierbarkeit einschätzen. Dabei gingen die Kinder überaus kreativ und selbständig vor. Für die Reflexion der gemachten Erfahrungen sowie das 
Auswählen der Bilder und Programmieren der Rallye standen zwei weitere Termine zur Verfügung. Zum Einsatz kamen mehrere Endgeräte (Tablets, Laptops, Smartphones) im Sinne einer bring-your-own-device-Praxis. Es zeigte sich dabei, dass eine kleinschrittige Planung der Rallye sinnvoll ist, für ein tiefergehendes Verstehen des gesamten Programmier-Prozesses. Auf diese Weise konnte ein aktiv-wahrnehmendes Anwenden und interaktives Nutzen der Medien sichergestellt werden.
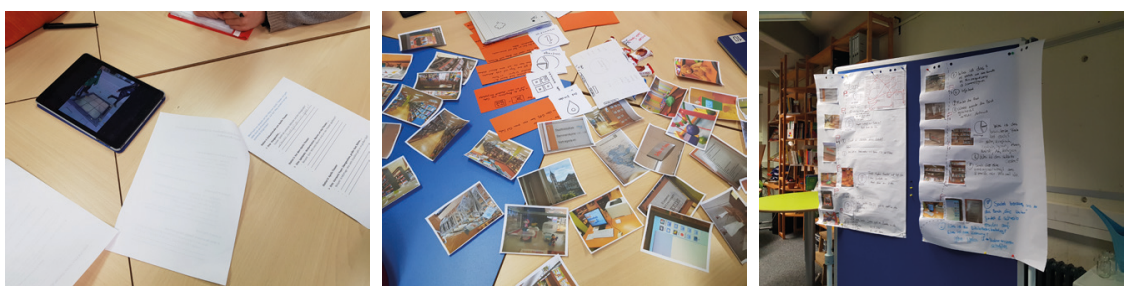

Abb. 4.: Didaktisches Material zur Vorbereitung für die Programmierung in der App. Offline-Bearbeitung von Fragen und Festlegung der Rallye-Strecken mit ausgedruckten Bildern.

\section{Fazit und Ausblick}

Mit Blick auf das hier beschriebene Pilotprojekt «FutereLAB - Digitales Lernen» kann gesagt werden: Für die kleinen Kohorte, bestehend aus knapp 50 teilnehmenden Schülerinnen und Schüler sowie Studierenden, eröffneten sich neue Handlungsräume, in denen sie ihre Rolle als selbstaktive Akteurinnen und Akteure erweitern sowie dialogisch orientierte Beziehungsstrukturen aufbauen konnten.

Als herausfordernd bei der Planung und Durchführung der einzelnen Treffen stellte sich dabei für die Studierenden die Altersmischung heraus sowie der heterogene Kenntnisstand und eine gleichzeitige Beteiligung aller Schülerinnen und Schüler. Bei der Programmierung waren regelmässige Pausen notwendig, um einem Konzentrations- und Motivationsverlust bei den Kindern entgegenzuwirken. Die Möglichkeit, sehr frei und eigenständig zu planen, wurde zunächst ebenfalls als herausfordernd von den Studierenden empfunden. Im Prozessverlauf änderte sich die Haltung diesbezüglich. Das selbständige Arbeiten im Team wurde im Nachhinein von den Studierenden als sehr gute Chance wahrgenommen, die 
eigene Handlungspraxis zu hinterfragen und konkreter auf den jeweiligen Kenntnisstand der Schülerinnen und Schüler einzugehen; auf diese Weise konnten sie situative Handlungskompetenz anbahnen. Ferner wurden das bewusste Erkunden eines unbekannten Ortes, das visuelle Vorbereiten und die Freiräume für Diskussionen innerhalb der Kleingruppen als sehr hilfreich eingeschätzt. Dies ermöglichte die Reflexion von Grenzen und Risiken der unmittelbaren und öffentlichen kommunikativen Handlungen.

Die Schülerinnen und Schüler zeigten eine sehr hohe Bereitschaft, sich auf Neues einzulassen. Dabei wendeten sie ihre bereits vorhandenen Handlungslogiken sicher an und schrieben diesen eigene Bedeutungen zu (vgl. Zinneker 2000). Eine Lehrerin äusserte hierzu mit Erstaunen, dass sie die Kinder in dem Lernsetting als überaus kompetent erlebt und auf diese Weise ganz neue Seiten an ihnen entdeckt habe. Aufgebaute Muster und Gewohnheiten konnten in das Neue integriert werden, wodurch sich Möglichkeiten für vielfältige Bildungsprozesse ergaben. Beispielsweise das ausbalancierte, dialogische Arbeiten mit den Studierenden in der Lernwerkstatt der Universität sowie mit Tablet, Smartphone und Laptop stellte eine Besonderheit dar, die die Kinder mit Spass, viel Freude und Motivation verbanden.

Alle Beteiligten konnten abseits von kommerziell betriebenen, digitalen Räumen Erfahrungen sammeln. Sowohl die 26 Studierenden als auch die 22 Schülerinnen und Schüler hatten die Möglichkeit, sich innerhalb eines bestimmten strukturellen Rahmens Wissen und Können anzueignen, um sachgemässes Umgehen mit digitalen Medien anzubahnen sowie ihre Vorkenntnisse in den Prozess einzubringen. Als hinderlich stellten sich dabei allzu negative Beurteilungen digitaler Medien heraus, die auf unreflektierten Theorien gründeten und das Nutzungsverhalten der Kinder auf naives Spielen reduzierten. Diese galt es zu hinterfragen. Förderlich mit Blick auf das Erschliessen von Räumen mit einem mobilen Endgerät hingegen wirkte das offene, kreative Lernsetting sowie die zur Verfügung gestellte Zeit und der Raum fürs Erkunden, Beobachten, Ausprobieren und Reflektieren. Dies ermöglichte allen Beteiligten ihre Wahrnehmungen und Vorstellungen über sich und ihre Umwelt zu entfalten - was als elementare Grundlage eines transformativen Bildungsprozesses für den Aufbau von Selbst- und Weltbildern dient. 
Abschliessend kann daher für die im Projekt involvierte Kohorte gesagt werden: Das dialogische Verständnis von Medienkompetenz und -bildung erscheint notwendig bei der Beschäftigung mit digitalen $\mathrm{Me}$ dien, weil sich beide am Projekt teilnehmenden Altersgruppen im Spannungsfeld zwischen werdend und seiend bewegen. Es ist anzunehmen, dass der angebahnte Prozess im Sinne eines transformativen Bildungsverständnisses auf eine tiefere Ebene im Subjekt abzielt und an keine messbaren Standards gebunden werden kann. Um diese Annahme jedoch zu konkretisieren und zu analysieren, sollten im Rahmen einer Folgestudie Denkstrukturen schulischer Akteurinnen und Akteure beim Lernen mit digitalen Medien u.a. zu folgenden forschungsrelevanten Fragestellungen erhoben werden: Welche Vorstellungen vom Sachgegenstand und Handlungsfeld bringen Lehramtsstudierende mit? Inwieweit verändern sich diese Vorstellungen im Handlungsfeld im Hinblick auf Kompetenzerwerb, Lehrerprofessionalisierung und digitale Technologien? Welches Verständnis von digitalen Technologien bringen Kinder mit? Welche Kompetenzen im Umgang mit digitalen Medien können realistisch in der Grundschule angebahnt werden?

\section{Literatur}

Altrichter, Herbert, Peter Posch, und Harald Spann. 2018. Lehrerinnen und Lehrer erforschen ihren Unterricht. 5., grundlegend überarbeitete Auflage. utb Schulpädagogik 4754. Bad Heilbrunn: Verlag Julius Klinkhardt.

Aufenanger, Stefan. 2015. «Wie die neuen Medien Kindheit verändern. Kommunikative, soziale und kognitive Einflüsse der Mediennutzung». merz | medien + erziehung 59 (2): 10-16.

Baacke, Dieter. 1998. «Medienkompetenz im Spannungsfeld von Handlungskompetenz und Wahrnehmungskompetenz». Vortrag, Pädagogische Hochschule Ludwigsburg, Dezember 8. https://www.ph-ludwigsburg.de/fileadmin/ subsites//lb-mpxx-t-ol/user_files/Baacke.pdf.

Baar, Robert, und Gudrun Schönknecht. 2018. Außerschulische Lernorte: didaktische und methodische Grundlagen. 1. Auflage. Reihe «Bildungswissen Lehramt», Band 30. Weinheim Basel: Beltz. 
Deutsche Gesellschaft für Erziehungswissenschaft, und Julia Koller. 2018. «Effekte von Verräumlichung durch digitale Medien in der wissenschaftlichen Weiterbildung. Real - virtuell - kontextuell». In Räume für Bildung - Räume der Bildung: Beiträge zum 25. Kongress der Deutschen Gesellschaft für Erziehungswissenschaft, herausgegeben von Edith Glaser, Hans-Christoph Koller, Werner Thole, und Salome Krumme, 274-82. Schriften der Deutschen Gesellschaft für Erziehungswissenschaft (DGfE). Opladen Berlin Toronto: Verlag Barbara Budrich.

Döbeli Honegger, Beat. 2016. Mehr als $\mathrm{O}$ und 1: Schule in einer digitalisierten Welt. 1. Auflage. Bern: hep, der Bildungsverlag.

Feierabend, Sabine, Theresa Plankenhorn, und Thomas Rathgeb. 2016. «JIM 2016. Jugend, Information, (Multi-) Media. Basisstudie zum Medienumgang 12bis 19-Jähriger in Deutschland». Herausgegeben von Medienpädagogischer Forschungsverbund Südwest. Medienpädagogischer Forschungsverbund Südwest. http://www.mpfs.de/fileadmin/files/Studien/JIM/2016/JIM_Studie_2016.pdf.

Feierabend, Sabine, Theresa Plankenhorn, und Thomas Rathgeb. 2017. «KIMStudie 2016. Kindheit, Internet, Medien. Basisstudie zum Medienumgang 6bis 13-Jähriger in Deutschland». Herausgegeben von Medienpädagogischer Forschungsverbund Südwest. Medienpädagogischer Forschungsverbund Südwest. http://www.mpfs.de/fileadmin/files/Studien/KIM/2016/KIM_2O16_ Web-PDF.pdf.

Foucault, Michel. 1990. «Andere Räume». In Aisthesis: Wahrnehmung heute oder Perspektiven einer anderen Ästhetik: Essais, herausgegeben von Karlheinz Barck, Peter Gente, Heidi Paris, und Stefan Richter, 1. Aufl, 34-46. ReclamBibliothek, Bd. 1352. Leipzig: Reclam.

Gesellschaft für Didaktik des Sachunterrichts, Hrsg. 2002. Perspektivrahmen Sachunterricht. Bad Heilbrunn/Obb: Klinkhardt. http://www.gdsu.de/wb/media/upload/pr_gdsu_2002.pdf.

Giddens, Anthony. 1988. Die Konstitution der Gesellschaft: Grundzüge e. Theorie d. Strukturierung. 1. Aufl. Theorie und Gesellschaft. Frankfurt/Main New York: Campus-Verl.

Hengst, Heinz. 2014. «Kinderwelten im Wandel». In Handbuch Kinder und Medien, herausgegeben von Angela Tillmann, Sandra Fleischer, und Kai-Uwe Hugger, 17-29. Wiesbaden: Springer Fachmedien Wiesbaden. https://doi. org/10.1007/978-3-531-18997-0_1.

Holzkamp, Klaus. 1995. Lernen: subjektwissenschaftliche Grundlegung. Studienausg. Frankfurt/Main New York: Campus-Verl.

Irion, Thomas. 2018. "Wozu digitale Medien in der Grundschule? Sollte das Thema Digitalisierung in Grundschulen tabuisiert werden?» Grundschule aktuell : Zeitschrift des Grundschulverbandes. Grundschulverband. http://nbn-resolving.org/urn:nbn:de:Oll1-pedocs-177129. 
Irion, Thomas. 2016. «Digitale Medienbildung in der Grundschule. Primarstufenspezische und medienpädagogische Anforderungen». In Neue Medien in der Grundschule 2.0. Grundlagen - Konzepte - Perspektiven, herausgegeben von Markus Peschel und Thomas Irion, 16-32. Frankfurt am Main: Grundschulverband e.V. http://nbn-resolving.org/urn:nbn:de:0111-pedocs-165589.

Kammerl, Rudolf. 2015. «Medienbildung - ein Bildungsangebot, bei dem Medienkompetenz hinten rauskommt?» Kinder- und Jugendschutz in Wissenschaft und Praxis, 3-7. http://www.kjug-zeitschrift.de/kjug-12015-prof-dr-rudolfkammerlmedienbildung-ein-bildungsangebot-bei-dem-medienkompetenzhinten-rauskommt/.

Kanwischer, Detlef, und Antje Schlottmann. 2017. «Virale Raumkonstruktionen Soziale Medien und Mündigkeit im Kontext gesellschaftswissenschaftlicher Medienbildung». Zeitschrift für Didaktik der Gesellschaftswissenschaften. Wochenschau Verlag 2: 60-78.

Klafki, Wolfgang. 1959. Studien zur Bildungstheorie und Didaktik. Weinheim: Beltz.

Klafki, Wolfgang. 2002. «Gesellschaftliche Funktionen und pädagogischer Auftrag der Schule in einer demokratischen Gesellschaft». In Schultheorie, Schulforschung und Schulentwicklung im politisch-gesellschaftlichen Kontext: ausgewählte Studien, herausgegeben von Barbara Koch-Priewe, Heinz Stübig, und Wilfried Hendricks, 41-62. Studien zur Schulpädagogik und Didaktik: [...], Reihe Pädagogik, Bd. 19. Weinheim Basel: Beltz.

KMK - Kultusministerkonferenz. 2016. «Bildung in der digitalen Welt. Strategie der Kultusministerkonferenz». Herausgegeben von Sekretariat der Kultusministerkonferenz. Kultusministerkonferenz. https://www.kmk.org/fileadmin/ Dateien/pdf/PresseUndAktuelles/2016/Bildung_digitale_Welt_Webversion. pdf.

Ladel, Silke, Julia Knopf, und Armin Weinberger, Hrsg. 2018. Digitalisierung und Bildung. Wiesbaden: Springer Fachmedien Wiesbaden. https://doi. org/10.1007/978-3-658-18333-2.

Löw, Martina. 2012. Raumsoziologie. 7. Aufl. Bd. 1506. Suhrkamp-Taschenbuch Wissenschaft. Frankfurt am Main : Suhrkamp.

Marotzki, Winfried, und Benjamin Jörissen. 2008. «Medienbildung». In Handbuch Medienpädagogik, herausgegeben von Uwe Sander, Friederike von Gross, und Kai-Uwe Hugger, 100-109. Wiesbaden: VS Verlag für Sozialwissenschaften. https://doi.org/10.1007/978-3-531-91158-8_11.

Moser, Heinz. 2019. Einführung in die Medienpädagogik: Aufwachsen im digitalen Zeitalter. Wiesbaden: Springer Fachmedien Wiesbaden. https://doi. org/10.1007/978-3-658-23208-5.

Orwell, George. 1993. 1984. 10. Aufl. Ullstein, Nr. 20456. Frankfurt/M Berlin: Ullstein. 
Peschel, Markus. 2016. «Medienlernen im Sachunterricht - Lernen mit Medien und Lernen über Medien». In Neue Medien in der Grundschule 2.0: Grundlagen - Konzepte - Perspektiven, herausgegeben von Markus Peschel und Thomas Irion, 141:33-49. Beiträge zur Reform der Grundschule. Frankfurt am Main: Grundschulverband e.V.

Piaget, Jean. 1981. Einführung in die genetische Erkenntnistheorie. 2. Aufl. Suhrkamp Taschenbuch, Wissenschaft 6. Frankfurt am Main: Suhrkamp.

Piaget, Jean. 1982. «Das In-Beziehung-Setzen der Perspektiven». In Perspektivenübernahme und soziales Handeln: Texte zur sozial-kognitiven Entwicklung, herausgegeben von Dieter Geulen, 1. Aufl, 75-85. Suhrkamp-Taschenbuch Wissenschaft 348. Frankfurt am Main: Suhrkamp.

Pirnay-Dummer, Pablo, und Andreas Spengler. 2019. «Medien im Unterricht». In Handbuch Schulpädagogik, herausgegeben von Marius Harring, Carsten Rohlfs, und Michaela Gläser-Zikuda, 483-95. UTB Schulpädagogik 8698. Münster New York: Waxmann.

Prensky, Marc. 2001. «Digital Natives, Digital Immigrants». On the Horizon, MCB University Press, 9 (5): 1-6. https://doi.org/10.1108/10748120110424816.

Spanhel, Dieter. 2010. «Medienbildung statt Medienkompetenz? Zum Beitrag von Bernd Schorb (merz 5/og)». merz I medien + erziehung 54 (1) : 49-54.

Spanhel, Dieter. 2011. «Medienbildung als Grundbegriff der Medienpädagogik. Begriffliche Grundlagen für eine Theorie der Medienpädagogik». In Medienbildung und Medienkompetenz. Beiträge zu Schlüsselbegriffen der Medienpädagogik, herausgegeben von Heinz Moser, Petra Grell, und Horst Niesyto, 95-120. München: kopaed. https://doi.org/10.21240/mpaed/20/2011.09.15.X.

Spannagel, Christian. 2015. «Zehn Irrtümer zum Einsatz digitaler Medien in der Schule». LOG IN 181/182: 34-39.

Spitzer, Manfred. 2012. Digitale Demenz. Wie wir uns und unsere Kinder um den Verstand bringen. München: Droemer.

Tillmann, Angela, und Kai-Uwe Hugger. 2014. «Mediatisierte Kindheit - Aufwachsen in mediatisierten Lebenswelten». In Handbuch Kinder und Medien, herausgegeben von Angela Tillmann, Sandra Fleischer, und Kai-Uwe Hugger, 31-45. Wiesbaden: Springer Fachmedien Wiesbaden. https://doi.org/10.1007/978-3531-18997-0_2.

Tulodziecki, Gerhard. 2011. «Zur Entstehung und Entwicklung zentraler Begriffe bei der pädagogischen Auseinandersetzung mit Medien». Herausgegeben von Heinz Moser, Petra Grell, und Horst Niesyto. MedienPädagogik: Zeitschrift für Theorie und Praxis der Medienbildung 20 Medienbildung im Spannungsfeld medienpädagogischer Leitbegriffe sowie Medienbildung und Medienkompetenz. Beiträge zu Schlüsselbegriffen der Medienpädagogik. München: kopaed: 11-39. https://doi.org/10.21240/mpaed/20/2011.09.11.X.

Tulodziecki, Gerhard, und Bardo Herzig. 2010. Mediendidaktik. Bd. 2. Handbuch Medienpädagogik. München: kopaed. 
Tulodziecki, Gerhard, Bardo Herzig, und Silke Grafe. 2019. Medienbildung in Schule und Unterricht - Grundlagen und Beispiele. 2. Aufl. Bad Heilbrunn: Klinkhardt/ UTB.

Turkle, Sherry. 2017. Alone Together: Why We Expect More from Technology and Less from Each Other. Hachette Book Group.

Weinert, Franz E., Hrsg. 2002. Leistungsmessungen in Schulen. 2. Aufl. Beltz Pädagogik. Weinheim: Beltz.

Wiesemann, Jutta, Clemens Eisenmann, und Inka Fürtig. 2015. «Medienpraxis in der (frühen) Kindheit. Ethnografische Exploration des familiären Smartphonegebrauchs». merz wissenschaft 59 (6): 39-47.

Wiesemann, Jutta, und Inka Fürtig. 2018. «Kindheit zwischen Smartphone und pädagogischem Schulalltag». In Institutionalisierungen von Kindheit: Childhood Studies zwischen Soziologie und Erziehungswissenschaft, herausgegeben von Tanja Betz, Sabine Bollig, Magdalena Joos, und Sascha Neumann, 1. Auflage, 196-212. Kindheiten. Weinheim: Beltz Juventa.

Zinnecker, Jürgen. 2000. «Selbstsozialisation. Essay über ein aktuelles Konzept». ZSE: Zeitschrift für Soziologie der Erziehung und Sozialisation 20 (3): 272-90. http://nbn-resolving.de/urn:nbn:de:011l-pedocs-109373. 